\title{
$034 \quad$ HIGHER THROMBUS BURDEN IN PATIENTS WITH SUBCLINICAL HYPOTHYROIDISM AFTER NON ST ELEVATION ACUTE CORONARY SYNDROME
}

K Balasubramaniam, ${ }^{1}$ G V Viswanathan, ${ }^{1}$ S M Marshall, ${ }^{1} \mathrm{~J} J$ Badimon, ${ }^{2}$ S Razvi, ${ }^{3}$ A G Zaman ${ }^{4}$ Newcastle University; ${ }^{2}$ Cardiovascular Institute, Mount Sinai School of Medicine; ${ }^{3}$ Gateshead Health NHS Foundation Trust; ${ }^{4}$ Freeman Hospital, Newcastle University

doi:10.1136/heartjnl-2013-304019.34

Introduction After an acute coronary syndrome, presence of subclinical hypothyroidism $(\mathrm{SCH})$ has been associated with increased risk of further thrombotic events. We aimed to evaluate platelet dependent thrombus (PDT) formation in patients after non-ST elevation acute coronary syndrome (NSTE-ACS) in relation to their thyroid status.

Methods We studied 70 patients 1 week after troponin positive NSTE-ACS. They were all treated according to current AHA/ ESC guidelines including $300 \mathrm{mg}$ loading dose followed by $75 \mathrm{mg}$ each of aspirin and clopidogrel. Patients with known thyroid disease were excluded. All patients underwent ex-vivo Badimon chamber assay to assess PDT. TSH, FT4 and FT3 were measured in all individuals. Those with TSH $>4.0 \mathrm{mU} / \mathrm{l}$ and normal FT4 were classed as having SCH $(n=12)$ whereas individuals with normal TSH and FT4 were classed as being euthyroid $(\mathrm{n}=58)$.

Results Baseline characteristics like age, gender, BMI, history of diabetes mellitus, blood pressure and previous vascular disease were similar between the groups. The SCH group had increased PDT (thrombus area $\mu^{2} / \mathrm{mm}$ : mean \pm SD, $23608 \pm 10498$ vs $16661 \pm 10902, p=0.02)$ when compared to the euthyroid group. Serum TSH ( $\beta$ co-efficient 0.28 ) and presence of diabetes ( $\beta$ co-efficient 0.29 ) were the only independent predictors of PDT in multiple regression analysis $(p<0.005)$.

Conclusions Patients after NSTE-ACS demonstrated increased thrombus formation in the presence of $\mathrm{SCH}$ despite optimal recommended secondary prevention therapy. This may explain the increased risk of further thrombotic events. Future trials need to be performed to assess the effect of individualised anti-thrombotic as well as thyroid hormone replacement therapy to reduce atherothrombotic risk in this population.

$\mathrm{SCH}$

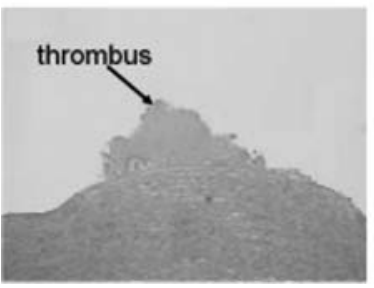

Euthyroid

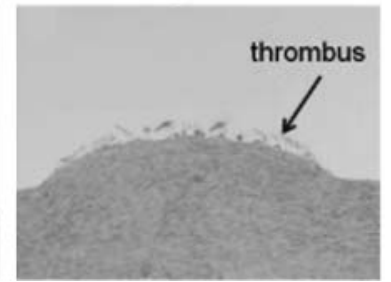

Figure 1 Post NSTE-ACS presence of SCH is associated with Higher thrombus formation when compared to euthyroid status. 